\title{
Análisis del efecto chimenea en fachadas ventiladas opacas mediante correlaciones del flujo másico inducido. Aplicación para el dimensionado de anchos de cámara
}

\author{
Stack effect analysis in opaque ventilated facades using induced mass flow \\ correlations. Application to the dimensioning of the chamber width \\ C. Suárez $^{(*)}$, J. L. Molina ${ }^{(*)}$
}

\section{RESUMEN}

En el presente artículo, se realiza un análisis general del problema del efecto chimenea aplicado a las fachadas ventiladas opacas. Desde un punto de vista energético, el diseño y la optimización de las fachadas ventiladas requiere un profundo conocimiento de los fenómenos térmicos y fluidodinámicos que se producen en la cámara ventilada y debido a la complejidad de estos fenómenos, el diseño óptimo supone un reto para arquitectos e ingenieros. Como resultado principal, se proponen unas correlaciones útiles para el cálculo del flujo másico inducido en la cavidad ventilada en función de la geometría y de la diferencia de temperaturas. El conjunto de casos de aplicación de las correlaciones cubre el rango habitual de interés en fachadas ventiladas opacas. Asimismo se realiza un análisis de sensibilidad del caudal inducido al variar los parámetros señalados.

Palabras clave: Fachadas ventiladas; efecto chimenea; CFD; caudal másico inducido.

\begin{abstract}
A general analysis of the stack effect problem applied to opaque ventilated facades is made in this paper. From an energetic point of view, the design and optimization of ventilated facades, require a deep understanding of the thermal and fluid dynamic phenomena that take place in the air gap, and, due to the complexity of these phenomena, the optimal design is a challenge to architects and engineers. As a main result, useful correlations for calculating the induced mass flow into the ventilated cavity are proposed depending on the geometry and the temperature difference. The set of application of the correlation covers the usual range of interest in opaque ventilated facades. It is also made a sensitive analysis of the induced flow with the indicated parameters.
\end{abstract}

Keywords: Ventilated facades; stack effect; CFD; induced mass flow.

(*) Escuela Superior de Ingenieros - Universidad de Sevilla (España).

Persona de contacto/Corresponding author: chss@us.es (Ch. Suárez)

Cómo citar este artículo/Citation: Suárez, C., Molina, J. L. (2015). Análisis del efecto chimenea en fachadas ventiladas opacas mediante correlaciones del flujo másico inducido. Aplicación para el dimensionado de anchos de cámara. Informes de la Construcción, 67(538): eo87, doi: http://dx.doi.org/10.3989/ic.13.155.

Licencia/License: Salvo indicación contraria, todos los contenidos de la edición electrónica de Informes de la Construcción se distribuyen bajo una licencia de uso y distribución Creative Commons Reconocimiento no Comercial 3.o. España (cc-by-nc). 


\section{INTRODUCCIÓN}

La fachada ventilada es una solución constructiva en auge. Además de las ventajas de su comportamiento térmico y acústico en comparación con un cerramiento convencional de una única hoja, las fachadas ventiladas presentan otra serie de virtudes como por ejemplo sus buenas prestaciones frente a problemas de humedad o su elevada calidad estética con gran diversidad de tipos de materiales y acabados, por citar algunas de ellas.

Podemos definir una fachada ventilada como un cerramiento convencional revestido exteriormente por otro de modo que entre ambos queda una cámara ventilada a la que el aire exterior puede acceder libremente. En su movimiento a través del canal, el aire accede al mismo por la zona inferior y sale por la zona superior.

La cámara ventilada crea un «efecto chimenea» provocado por el calentamiento de la hoja exterior, de modo que se produce una variación de la densidad del aire en el interior de la cámara con respecto al aire ambiente, con el consiguiente movimiento ascendente por convección natural. En la Figura 1 se muestra un esquema en el que se plantea el problema de convección natural en una fachada ventilada opaca de ancho b y alto L. Debido a la diferencia de temperaturas entre las superficies interiores del canal $\mathrm{T}_{\mathrm{h}} \mathrm{y}$ el aire exterior $\mathrm{T}_{\mathrm{o}}$, bajo la acción de la gravedad $\mathrm{g}$, se establece un flujo másico inducido $\mathrm{q}_{\mathrm{m}}$ por convección natural.

A efectos del presente trabajo se considera que la contribución al flujo de aire a través de las juntas que separan los distintos módulos de la fachada ventilada (en el caso de que existieran) es despreciable frente al caudal que se establece en la propia cámara ventilada. Este movimiento ascendente del aire en el interior de la cámara ventilada juega un papel decisivo en el comportamiento térmico-energético del elemento, ya que de las características de este movimiento depende el flujo de calor que entra al edificio a través de la hoja interior.

Respecto al comportamiento térmico, la fachada ventilada presenta ventajas en comparación con una fachada convencional. Durante los meses de verano, la hoja exterior bloquea la radiación solar por lo que disminuye la temperatura superficial de la hoja interior. Además el flujo másico inducido contribuye a evacuar el calor en la cámara, siendo esperable una disminución en el flujo de calor que entra al edificio. Gagliano et al. (1) estiman que esta reducción del flujo de calor es superior al $40 \%$ en comparación con la misma fachada sin ventilar. Este comportamiento energético es menos ventajoso para valores bajos de radiación solar (por ejemplo para fachadas de orientación norte). Por otra parte, en los meses de invierno, con una radiación solar incidente mucho menor que en verano, la temperatura exterior y la temperatura en el interior de la cámara son aproximadamente iguales y el efecto chimenea es de menor cuantía. El movimiento de aire permite la evacuación del vapor de agua, disminuyendo la posibilidad de condensaciones intersticiales. Reforzando lo anterior, son muy ilustrativos los resultados obtenidos mediante simulación térmica de edificios por Irulegi (2), en los que se cuantifican los niveles de ahorro en edificios de oficinas hasta valores superiores al $40 \%$ en muchos casos en términos de demanda de calefacción (dependiendo de la zona climática y de la geometría del edificio).

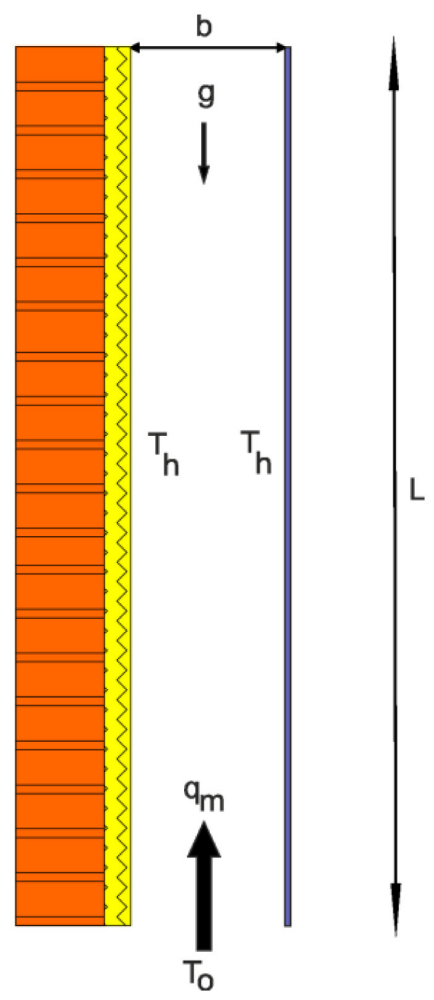

Figura 1. Problema de convección natural en una fachada ventilada opaca.

En el caso de que por convección natural no se obtenga el nivel de ventilación deseado en la cámara ventilada, existe la posibilidad de forzar el movimiento mediante el uso de ventiladores (convección forzada). En ambos casos, ya sea por convección natural o por convección forzada, existen aplicaciones en las que puede emplearse el caudal de aire que se establece en la cámara para ventilar el edificio. Así, en los meses de invierno, la cámara ventilada serviría como precalentador del aire antes de la alimentación al sistema de ventilación, por lo que se conseguiría una disminución del consumo de calefacción.

Los parámetros que determinan el caudal másico qm que se induce por convección natural son el ancho b y el alto L de la cámara y la diferencia de temperaturas $\Delta \mathrm{T}$ entre el aire exterior y las superficies interiores de las paredes de la cámara. El alto y ancho son parámetros geométricos de diseño que podrían optimizarse para conseguir un mayor caudal de ventilación. La diferencia de temperaturas viene principalmente dada por las condiciones climáticas (temperatura y radiación solar) que haya en la ubicación/orientación de la fachada ventilada, pero también influyen las características constructivas a través del nivel de la transmitancia térmica y de la absortividad. Así, en una fachada ventilada sobre la que incide un nivel de radiación solar dado, con una hoja exterior metálica se obtienen mayores diferencias de temperatura en comparación con una hoja exterior cerámica, al ser mayor la conductividad térmica del metal y consiguientemente la transmitancia térmica. Del mismo modo, con materiales de mayores absortividades en la hoja exterior se obtienen mayores diferencias de temperaturas.

A pesar de la innegable importancia del efecto chimenea para el cálculo del comportamiento térmico-energético del elemento, existen pocos modelos térmicos que resuelvan con 
el suficiente detalle el problema de convección natural que tiene lugar en la cavidad de la fachada ventilada. Entre estos modelos, cabe destacar el de Balocco (3), el de Marinosci (4) y el de Ruiz-Pardo (5). Balocco (3) define un modelo completo del elemento fachada ventilada. A cada volumen de control, se le aplica un balance de energía en régimen permanente y las ecuaciones se resuelven iterativamente mediante un procedimiento basado en un código de elementos finitos. Marinosci (4) realiza una investigación experimental y numérica del comportamiento térmico de una fachada ventilada. Para ello emplea un edificio de prueba usando el programa ESP-r, cuyo código está basado en técnicas de volúmenes finitos. Los coeficientes convectivos se calculan a partir de las correlaciones de Alamdari-Hammond, que dependen únicamente de la diferencia de temperaturas entre las superficies del canal y el ambiente y la altura del elemento. Ruiz-Pardo (5), desarrolla un modelo térmico en estado transitorio de una fachada ventilada basado en el método de las diferencias finitas. Los coeficientes convectivos en el interior del canal se calculan empleando las correlaciones de Sparrow, que dependen de la relación de aspecto del canal, el número de Rayleigh y la altura del canal. Estas diferentes hipótesis de partida en los modelos, especialmente las relacionadas con el problema del movimiento del aire en la cavidad ventilada, crean incertidumbre en los resultados que afectan a aspectos críticos como las temperaturas superficiales de los cerramientos y el flujo másico inducido.

Para suplir esta incertidumbre, se propone un estudio termofluidodinámico detallado del canal ventilado que permite el cálculo del flujo másico inducido, de gran utilidad para la evaluación del comportamiento térmico de las fachadas ventiladas.

En el presente artículo, basado en los resultados de la tesis doctoral (6), se realiza un análisis general del problema del efecto chimenea aplicado a las fachadas ventiladas opacas y como resultado principal, se proponen unas correlaciones útiles para el cálculo del flujo másico inducido en la cavidad ventilada en función de la geometría y de la diferencia de temperaturas. Asimismo se analiza la influencia que tienen sobre este caudal inducido variaciones de la geometría del elemento y de la diferencia de temperaturas entre el aire exterior y las superficies interiores de la cavidad ventilada.

\section{METODOLOGÍA}

El estudio del problema del movimiento del aire en el interior de una fachada ventilada se realiza mediante técnicas de computación fluidodinámica (CFD), utilizando como herramienta el software Fluent ${ }^{\odot}$. Para resolver el problema, se desarrolla un modelo adecuado del elemento y se parte de una serie de hipótesis que hacen que el problema sea resoluble por el motor de cálculo del software, que resuelve en todos los puntos del dominio las ecuaciones de Navier-Stokes. Las ecuaciones de masa, momento y energía, asociadas a sus condiciones de contorno, se resuelven de manera iterativa hasta la convergencia de los residuos de las variables de interés (velocidades, temperaturas, flujo másico, etc.). El flujo se considera en régimen permanente y laminar y las propiedades del aire se asumen constantes con excepción de la densidad en la que se supone que se rige por la ley de los gases ideales.

Dado que las condiciones de velocidad y presión del aire en la entrada del canal son desconocidas, fue preciso considerar un dominio computacional de cálculo extenso en el que las condiciones de contorno no se impusieron directamente en la entrada y la salida del canal sino en la frontera de dicho de dominio computacional donde la presión y velocidad sí son conocidas y pueden suponerse iguales a las condiciones ambientales. Esta técnica es de uso habitual en cálculos numéricos mediante CFD y ha sido empleada por diversos autores. Por citar algunos ejemplos, Kheireddinein (7), Kettleborough et al. (8), Nakamura et al. (9) and Naylor et al. (10) Chang and Lin (11), Ramanathan and Kumar (12), Shy et al. (13), Morrone et al. (14) y Campo et al. (15) han considerado en sus estudios numéricos dominios computacionales extensos para el análisis de la convección natural en un canal ventilado. La región computacional se extiende fuera de los límites del canal en todas las direcciones, dejando la zona de interés de estudio en el centro del dominio. En el interior del canal ventilado, que es donde se pretende estudiar en detalle el movimiento del aire, se realizará un mallado especialmente fino con el fin de obtener resultados lo más precisos posible.

En la siguiente figura se muestran las condiciones de contorno del problema (ver Figura 2). En los límites del dominio computacional (de tamaño $\mathrm{b}_{\mathrm{dom}} \times \mathrm{L}_{\mathrm{dom}}$ ) se considera que el aire tiene una velocidad nula y que la temperatura del aire es la del ambiente. Todas las superficies se consideran adiabáticas excepto las superficies interiores del canal que se suponen isotermas.

Desde el punto de vista de la mecánica de fluidos, el número adimensional asociado con la transferencia de calor en problemas de convección natural es el Número de Rayleigh ( $\mathrm{Ra}$ ). En el caso particular de la geometría de estudio, es usual el empleo del número de Rayleigh basado en el ancho del canal b como longitud característica y modificado por la relación del aspecto del canal, $\mathrm{Ra}_{\mathrm{b}}^{*}$

$$
\mathrm{Ra}_{\mathrm{b}}^{*}=\mathrm{Ra}_{\mathrm{b}} \cdot \frac{\mathrm{b}}{\mathrm{L}}=\frac{\mathrm{g} \cdot \beta \cdot \Delta \mathrm{T} \cdot \mathrm{b}^{3}}{\mathrm{v} \cdot \alpha} \cdot \frac{\mathrm{b}}{\mathrm{L}}
$$

Donde:

b ancho del canal (m)

$\mathrm{L} \quad$ alto del canal (m)

$\mathrm{T}_{\mathrm{h}}, \mathrm{T}_{\mathrm{o}}$ temperaturas superficial y exterior respectivamente $\left({ }^{\circ} \mathrm{C}\right)$

$\Delta \mathrm{T} \quad$ diferencia de temperaturas $\left({ }^{\circ} \mathrm{C}\right), \Delta \mathrm{T}=\mathrm{T}_{\mathrm{h}}-\mathrm{T}_{\mathrm{o}}$

g $\quad \operatorname{gravedad}\left(\mathrm{m} / \mathrm{s}^{2}\right)$

$\beta \quad$ coeficiente de dilatación a presión constante $(1 / \mathrm{K})$

$v \quad$ viscosidad cinemática $\left(\mathrm{m}^{2} / \mathrm{s}\right)$

a difusividad térmica $\left(\mathrm{m}^{2} / \mathrm{s}\right)$

En cuanto al flujo másico inducido en problemas de convección natural, es útil el uso de una variable denominada flujo másico adimensional $\mathrm{M}$, que se correlaciona apropiadamente con el número de Rayleigh y está definido por la siguiente expresión:

$$
M=\frac{q_{m}}{\rho \cdot \alpha \cdot R a_{b}}
$$

Donde:

$\mathrm{q}_{\mathrm{m}} \quad$ flujo másico dimensional por unidad de profundidad ( $\mathrm{kg} / \mathrm{s} \mathrm{m})$

$\rho \quad$ densidad $\left(\mathrm{kg} / \mathrm{m}^{3}\right)$

a difusividad térmica $\left(\mathrm{m}^{2} / \mathrm{s}\right)$

$\mathrm{Ra}_{\mathrm{b}} \quad$ número de Rayleigh basado en el ancho del canal b 


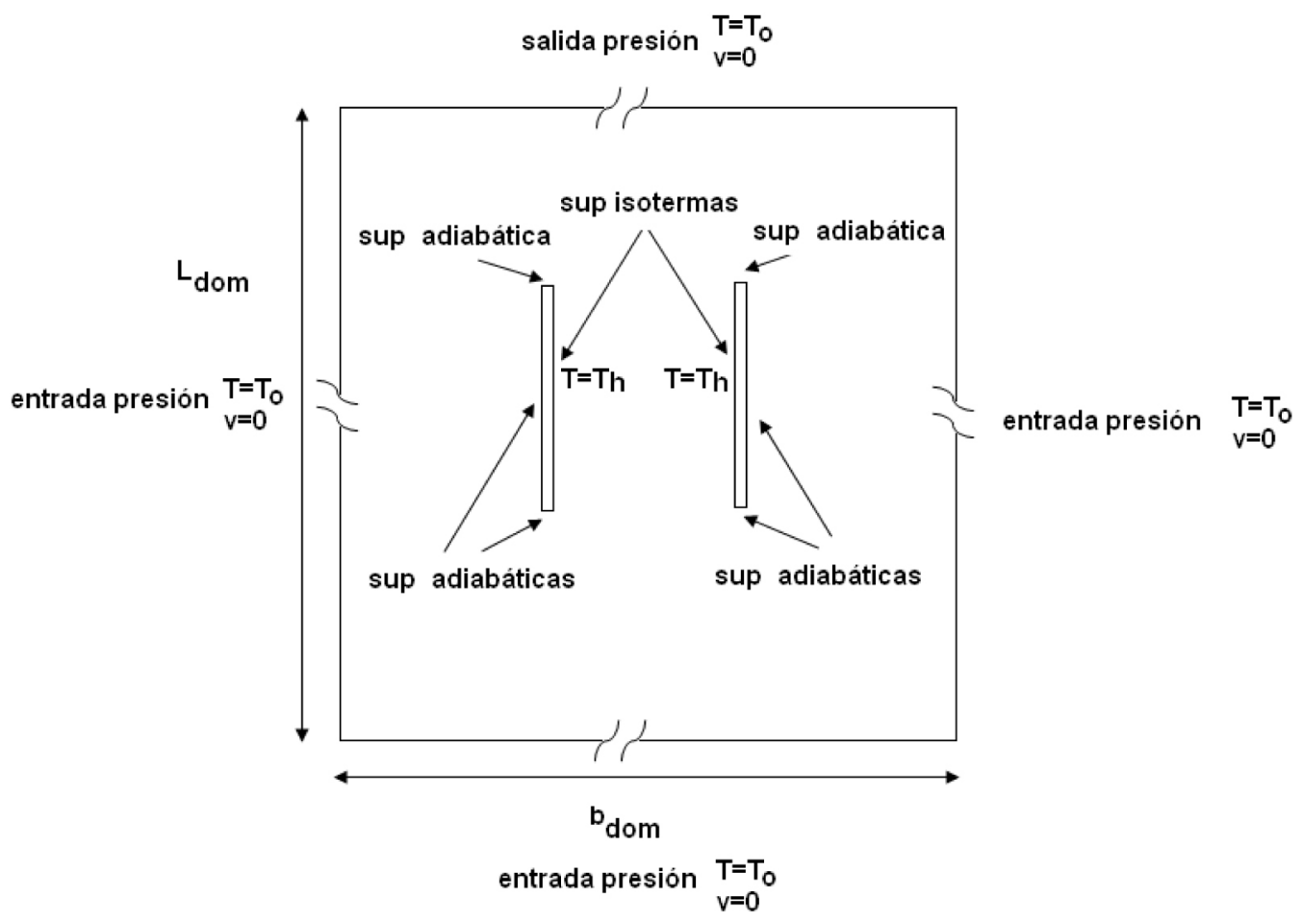

Figura 2. Dominio computacional y condiciones de contorno del problema.

Para la geometría del canal ventilado se tienen dos situaciones típicas con respecto al perfil de velocidades que se establece por convección natural y que están directamente relacionadas con el valor del número de Rayleigh modificado $\mathrm{Ra}_{\mathrm{b}}{ }_{\mathrm{b}}$. Por una parte, para valores bajos de $\mathrm{Ra}_{\mathrm{b}}{ }^{*}$ (asociado a anchos de canal y diferencias de temperatura pequeños), se tiene que la conducción es dominante frente a la convección y que el flujo másico inducido es pequeño. En este caso, el perfil de velocidades en el interior de la fachada ventilada es típicamente parabólico. Por otra parte, para valores mayores de $\mathrm{Ra}_{\mathrm{b}}{ }^{*}$ (asociado a anchos de canal y diferencias de temperatura mayores) se tiene que la convección es dominante frente a la conducción y que el flujo másico inducido es mayor. En este segundo caso, el perfil de velocidades en el interior de la fachada ventilada alcanza máximos relativos en las proximidades de las paredes y un mínimo relativo en el eje central del canal (ver Figura 3).

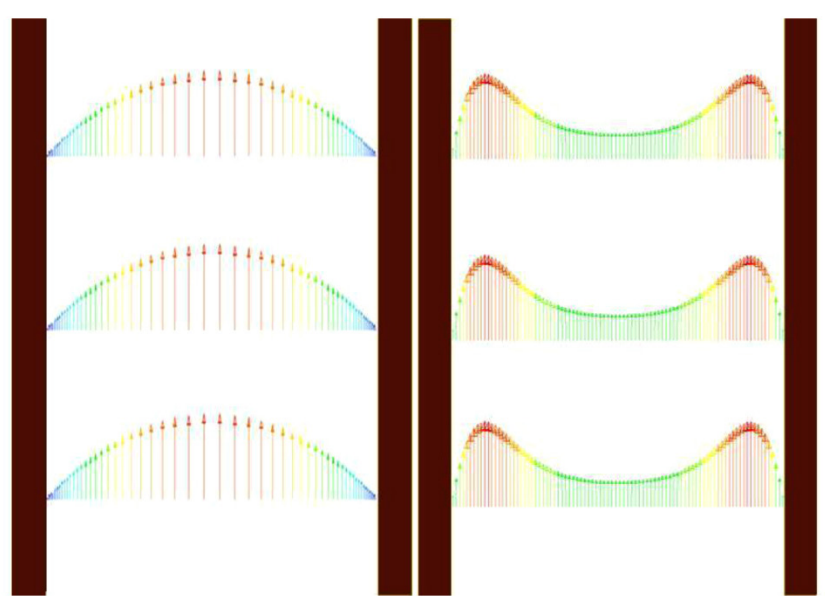

Figura 3. Perfiles de velocidad típicos, bajos $\mathrm{Ra}_{\mathrm{b}}{ }^{*}$ (izquierda), altos $\mathrm{Ra}_{\mathrm{b}}{ }^{*}$ (derecha).

\section{CORRELACIÓN OBTENIDA Y VALIDACIÓN DE LOS RESULTADOS}

Para obtener una solución válida del problema los resultados obtenidos de las variables de interés no pueden depender ni del tamaño del dominio computacional ni del nivel de mallado. El mallado se realiza de manera que en la zona de interés (el canal ventilado) se tengan un número suficiente de nodos, especialmente en las proximidades de las paredes donde son esperables los mayores gradientes de temperatura y de velocidades. Por ello, la distribución de los nodos elegida es mayor en las proximidades de las paredes, con un factor geométrico que dependiendo de los casos varía entre de 1.01 y 1.05. Asimismo, en dirección vertical se ha elegido una mayor densidad en las proximidades de la entrada y salida del canal, donde también son esperables mayores gradientes de velocidades y temperaturas (factor geométrico también en rango de 1.01 y 1.05, dependiendo de la geometría del caso simulado). En la Figura 4 se muestra a modo ilustrativo esta distribución de los nodos en el mallado para la zona inferior de la fachada ventilada.

Es necesario hacer un estudio previo de la independencia de los resultados con el tamaño del dominio computacional y también con el nivel de mallado. Los resultados de este análisis se muestran en la Tabla 1 para dos casos con valores de $\mathrm{Ra}_{\mathrm{b}}{ }^{*}$ de 80 y 7865, representativos de las situaciones características en el rango laminar en las que respectivamente domina la conducción y la convección.

En los casos 1-4 se mantuvo una densidad de mallado fija en el interior de la fachada ventilada (con 64 nodos en dirección horizontal y 200 nodos en dirección vertical) y se varió el tamaño del dominio computacional. Se observó gran influencia del tamaño del dominio computacional en los resultados del flujo másico inducido, por lo que fue necesario explorar tamaños mucho mayores al considerado inicialmente. Los resultados mostraron que a partir de un tamaño del dominio computacional 200 veces 


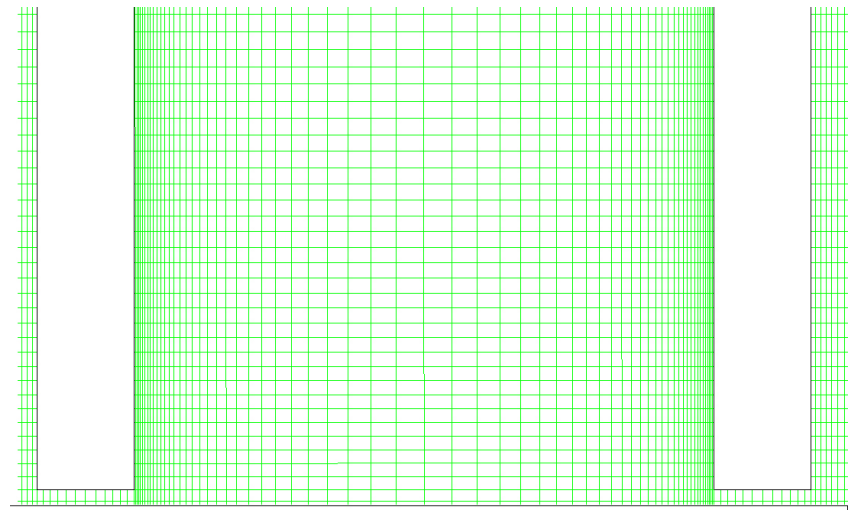

Figura 4. Mallado, zona inferior de la fachada ventilada.

mayor que el tamaño del elemento fachada ventilada (de ancho b y altura L), el resultado obtenido en términos del flujo másico inducido apenas varía. En los casos 4-6, se mantuvo un tamaño del dominio computacional fijo de 200 b $\times 200$ L y se exploró la influencia en los resultados de variar la densidad del mallado. Los resultados mostraron una menor influencia del número de nodos, siendo los resultados de los tres casos prácticamente iguales entre sí, con diferencias inferiores al $1 \%$. Las características del caso 5 con un tamaño computacional de $200 \mathrm{~b} \times 200 \mathrm{~L}$ y un nivel de mallado de $128 \times 200$ nodos en el interior del canal fueron las seleccionadas para realizar las simulaciones.

Una vez determinados el tamaño del dominio computacional y el nivel de mallado, se obtuvieron los flujos másicos inducidos en el canal en diferentes casos de interés con diferentes anchos de canal, alturas y diferencias de temperaturas. El conjunto de casos estudiados abarca fachadas ventiladas con valores típicos del número de Rayleigh modificado $\mathrm{Ra}_{\mathrm{b}}{ }^{*}$ inferiores a $10^{5}$, siendo el problema típicamente laminar. Para este conjunto de casos se obtuvieron unas correlaciones que permiten el cálculo del flujo másico a partir de los parámetros anteriormente señalados.

Las correlaciones seleccionadas para el flujo másico adimensional se muestran a continuación. Dado el distinto com- portamiento observado en los perfiles de velocidad dependiendo del valor del número de Rayleigh modificado $\mathrm{Ra}_{\mathrm{b}}{ }^{*}$, se presentan dos correlaciones en los dos tramos diferenciados: $10^{\circ} \leq \mathrm{Ra}_{\mathrm{b}}{ }^{*}<10^{2}$, en el que domina la conducción y $10^{2} \leq \mathrm{Ra}_{\mathrm{b}}{ }^{*}<10^{5}$ en la que domina la convección. De esta manera el análisis resulta coherente con las observaciones realizadas en los perfiles de velocidad y además se consigue una mayor precisión en los resultados.

En primer lugar se muestran los resultados en el rango $10^{\circ} \leq \mathrm{Ra}_{\mathrm{b}}{ }^{*}<10^{2}$, en el que la conducción es dominante. La función seleccionada para la correlación es un polinomio logarítmico de cuarto orden cuyos coeficientes se muestran en la Tabla 2.

$$
\mathrm{M}_{\text {isot }}=\mathrm{a}+\mathrm{b} \ln \left(\mathrm{Ra}_{\mathrm{b}}^{*}\right)+\mathrm{c} \ln \left(\mathrm{Ra}_{\mathrm{b}}^{*}\right)^{2}+\mathrm{d} \ln \left(\mathrm{Ra}_{\mathrm{b}}^{*}\right)^{3}+\mathrm{e} \ln \left(\mathrm{Ra}_{\mathrm{b}}^{*}\right)^{4}
$$

En segundo lugar se muestran los resultados en el rango $10^{2} \leq \mathrm{Ra}_{\mathrm{b}}{ }^{*}<10^{5}$, en el que la convección es dominante. La función elegida para la correlación es un polinomio logarítmico inverso de cuarto orden cuyos coeficientes se muestran en la Tabla 3.

$$
\mathrm{M}_{\text {isot }}=\mathrm{a}+\frac{\mathrm{b}}{\ln \left(\mathrm{Ra}_{\mathrm{b}}^{*}\right)}+\frac{\mathrm{c}}{\ln \left(\mathrm{Ra}_{\mathrm{b}}^{*}\right)^{2}}+\frac{\mathrm{d}}{\ln \left(\mathrm{Ra}_{\mathrm{b}}^{*}\right)^{3}}+\frac{\mathrm{e}}{\ln \left(\mathrm{Ra}_{\mathrm{b}}^{*}\right)^{4}}
$$

Una vez obtenido el flujo másico adimensional es inmediato obtener el valor del flujo másico dimensional por unidad de profundidad del canal:

$$
q_{m}=M \cdot \rho \cdot \alpha \cdot R a_{b}
$$

La concordancia de los resultados de la correlación propuesta con los resultados reportados por otros autores es excelente. Como puede apreciarse en la Figura 5 , los resultados se ajustan adecuadamente a la correlación obtenida por Ayinde (16) a partir de experimentos mediante mediciones del perfil de velocidades mediante un anemómetro láser Doppler y a los resultados reportados por Olsson (17).

Asimismo los resultados de la correlación propuesta se ajustan a los resultados de las simulaciones CFD realizadas en el presente trabajo, con un error medio del $2 \%$ y del $8 \%$ en

Tabla 1. Análisis de independencia de los resultados.

\begin{tabular}{|c|c|c|c|c|}
\hline Caso & Tamaño $\left(\mathbf{b}_{\text {dom }} \times \mathbf{L}_{\text {dom }}\right)$ & Nodos $\left({ }^{*}\right)$ & $\mathbf{q}_{\mathbf{m}}(\mathbf{g} / \mathbf{s}), \mathbf{R a}_{\mathbf{b}}{ }^{*}=\mathbf{8 0}$ & $\mathbf{q m}(\mathbf{g} / \mathbf{s}), \mathbf{R a}_{\mathbf{b}}{ }^{*}=\mathbf{7 8 6 5}$ \\
\hline 1 & $3 \mathrm{~b} \times 3 \mathrm{~L}$ & $64 \times 200$ & 0,84 & 6,31 \\
\hline 2 & $20 b \times 20 \mathrm{~L}$ & $64 \times 200$ & 1,05 & 9,03 \\
\hline 3 & $125 \mathrm{~b} \times 100 \mathrm{~L}$ & $64 \times 200$ & 1,25 & 9,37 \\
\hline 4 & $200 b \times 200 \mathrm{~L}$ & $64 \times 200$ & 1,29 & 9,55 \\
\hline 5 & $200 b \times 200 \mathrm{~L}$ & $128 \times 200$ & 1,28 & 9,54 \\
\hline 6 & $200 b \times 200 \mathrm{~L}$ & $128 \times 400$ & 1,27 & 9,53 \\
\hline
\end{tabular}

${ }^{*}$ Respectivamente número de nodos horizontales y verticales dentro del canal.

Tabla 2. Coeficientes de la correlación del flujo másico adimensional, $10^{\circ} \leq \mathrm{Ra}_{\mathrm{b}}{ }^{*}<10^{2}$.

\begin{tabular}{|c|c|c|c|c|c|}
\hline Rango & a & b & c & d & e \\
\hline $10^{\circ} \leq \mathrm{Ra}_{\mathrm{b}}{ }^{*}<10^{2}$ & $9.446 \mathrm{E}-\mathrm{O} 2$ & $-1.840 \mathrm{E}-03$ & $-3.388 \mathrm{E}-03$ & $4.281 \mathrm{E}-\mathrm{O} 4$ & $-4.297 \mathrm{E}-\mathrm{O} 5$ \\
\hline
\end{tabular}

Tabla 3. Coeficientes de la correlación del flujo másico adimensional, $10^{2} \leq \mathrm{Ra}_{\mathrm{b}}{ }^{*} \leq 10^{5}$.

\begin{tabular}{|c|c|c|c|c|c|}
\hline Rango & $\mathbf{a}$ & $\mathbf{b}$ & $\mathbf{c}$ & $\mathbf{d}$ & $\mathbf{e}$ \\
\hline $10^{2} \leq \mathrm{Ra}_{\mathrm{b}}{ }^{*} \leq 10^{5}$ & $-6.415 \mathrm{E}-\mathrm{O} 3$ & $2.873 \mathrm{E}-\mathrm{O} 1$ & $-5.472 \mathrm{E}+\mathrm{OO}$ & $4.374 \mathrm{E}+\mathrm{O} 1$ & $-9.334 \mathrm{E}+\mathrm{O} 1$ \\
\hline
\end{tabular}




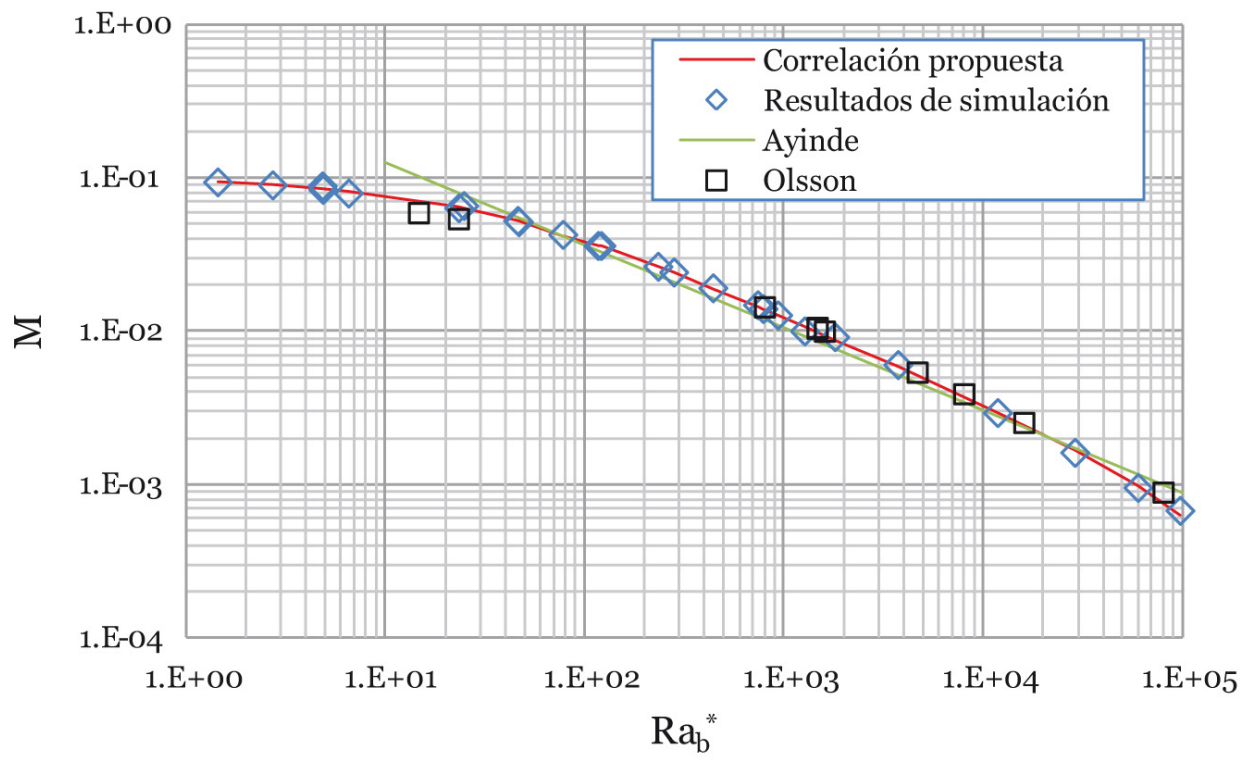

Figura 5. Comparación de los resultados de la correlación propuesta con los de otros autores.

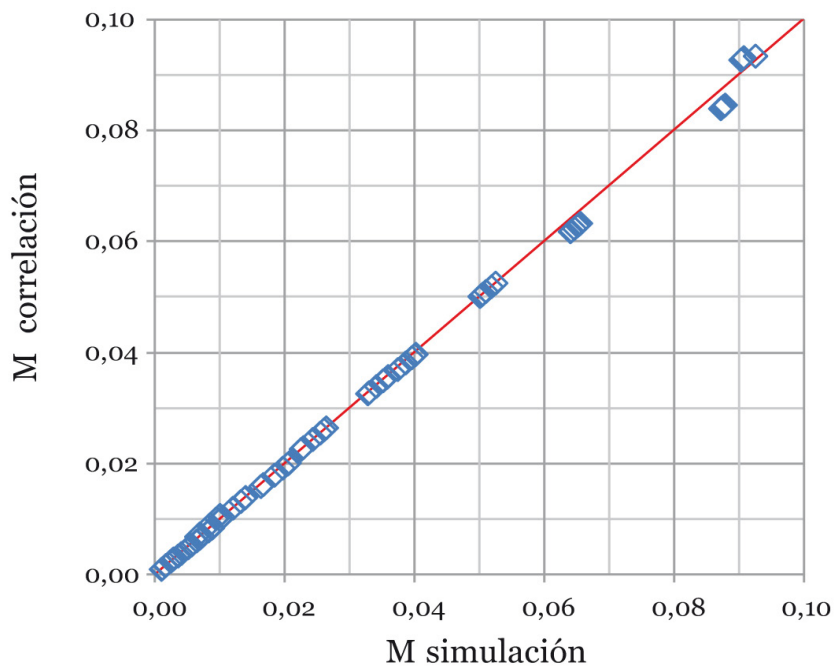

Figura 6. Comparación de los resultados de la correlación propuesta con los resultados de las simulaciones CFD.

el peor de los casos estudiados. En la Figura 6 se comparan ambos resultados. Se muestra como referencia la línea de $45^{\circ}$ que indicaría una coincidencia exacta entre los resultados dados por las simulaciones (eje de abscisas) y los resultados dados por la correlación (eje de ordenadas).

\section{ANÁLISIS DE SENSIBILIDAD}

Dada una fachada ventilada con un ancho b, un alto L y una diferencia de temperaturas $\Delta \mathrm{T}$ entre las superficies interiores del canal y el aire exterior dadas, se analiza a continuación el efecto que tiene sobre el caudal másico inducido un aumento de cada una de estas variables. Para el análisis se tiene en cuenta que, de acuerdo con la expresión [5], el flujo másico inducido $\mathrm{q}_{\mathrm{m}}$ es proporcional al flujo másico adimensional $\mathrm{M}$ y al número de Rayleigh basado en el ancho del canal $b, \mathrm{Ra}_{\mathrm{b}}$.

\section{Efecto del ancho $b$ de la fachada ventilada}

$\mathrm{Al}$ aumentar b se produce un doble efecto contrapuesto. Por una parte aumenta $\mathrm{Ra}_{\mathrm{b}}$ (factor al cubo), pero por otra parte, como se desprende de la Figura 5, se tiene que el flujo másico adimensional inducido $\mathrm{M}$ disminuye al aumentar el valor de $\mathrm{Ra}_{\mathrm{b}}{ }^{*}$. Por lo tanto no puede inferirse una relación directa entre el aumento del ancho del canal b y el flujo másico inducido $\mathrm{q}_{\mathrm{m}}$.

En la Figura 7 se muestra el caudal inducido para el caso de una fachada de $3 \mathrm{~m}$ de altura y diferentes valores del ancho del canal y de diferencias de temperaturas. Se aprecia que el aumento del ancho del canal, no implica necesariamente un aumento del caudal inducido. Así, aunque para diferencias de temperaturas de hasta $10^{\circ} \mathrm{C}$ se aprecia un aumento del caudal al aumentar el ancho del canal, para diferencias de temperaturas mayores se observa que existe un ancho del canal para el que se produce un máximo de caudal inducido (para $\Delta \mathrm{T}=20^{\circ} \mathrm{C}$ es $9 \mathrm{~cm}$ y para $\Delta \mathrm{T}=40^{\circ} \mathrm{C}$ es $8 \mathrm{~cm}$ ).

\section{Efecto del alto L de la fachada ventilada}

$\mathrm{Al}$ aumentar L se produce un aumento del caudal inducido. Dado que $\mathrm{Ra}_{\mathrm{b}}$ no depende de la altura del canal, en este caso se tiene que la altura del canal afecta únicamente al flujo

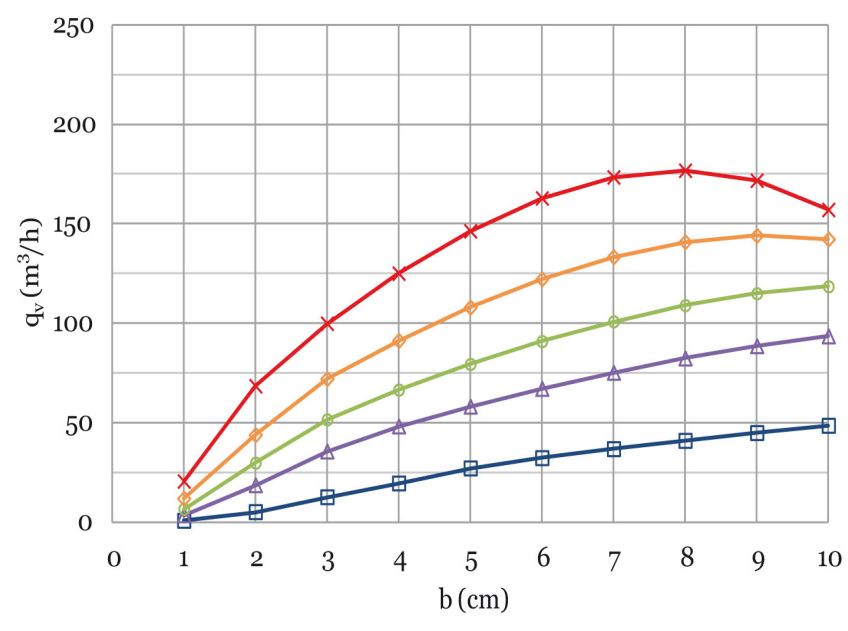

$\because \Delta \mathrm{T}=1^{\circ} \mathrm{C} \longrightarrow \Delta \mathrm{T}=5^{\circ} \mathrm{C} \smile \Delta \mathrm{T}=10^{\circ} \mathrm{C} \smile \Delta \mathrm{T}=20^{\circ} \mathrm{C} \nsucc \Delta \mathrm{T}=40^{\circ} \mathrm{C}$

Figura 7. Caudal volumétrico de aire inducido en función del ancho del canal para varias diferencias de temperatura $(\mathrm{L}=3 \mathrm{~m})$. 


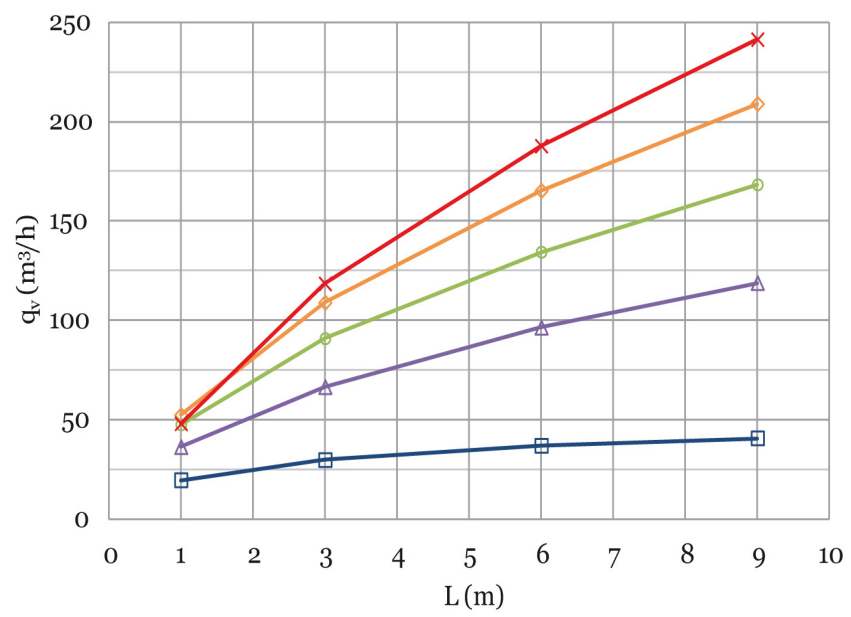

$\square \mathrm{b}=2 \mathrm{~cm} \triangle \mathrm{b}=4 \mathrm{~cm} \multimap \mathrm{b}=6 \mathrm{~cm} \leadsto \mathrm{b}=8 \mathrm{~cm} \leadsto \mathrm{b}=10 \mathrm{~cm}$

Figura 8. Caudal volumétrico de aire inducido en función del alto del canal para varios anchos de canal (DT $=10)$.

másico adimensional $\mathrm{M}$ a través del $\mathrm{Ra}_{\mathrm{b}}{ }^{*}$, de manera que al aumentar L disminuye $\mathrm{Ra}_{\mathrm{b}}{ }_{\mathrm{b}} \mathrm{y}$ y consecuentemente aumenta $\mathrm{M}$, como se desprende de la Figura 5. Por lo tanto en este caso sí puede inferirse que, fijados un ancho del canal y una diferencia de temperaturas un aumento de la altura de la fachada ventilada produce un aumento del caudal inducido $\mathrm{q}_{\mathrm{m}}$.

En la Figura 8 se muestra el caudal inducido para el caso de una fachada en la que se ha fijado una diferencia de temperaturas de $10^{\circ} \mathrm{C}$ para diferentes valores de la altura y del ancho del canal. Se aprecia que, en todo el rango de los valores del ancho del canal, un aumento de la altura implica un aumento del caudal inducido.

\section{Efecto de la diferencia de temperaturas $\Delta T$ de la fachada ventilada}

$\mathrm{Al}$ aumentar $\Delta \mathrm{T}$ se produce un doble efecto contrapuesto. Por una parte aumenta $\mathrm{Ra}_{\mathrm{b}}$, pero por otra parte se tiene que el flujo másico adimensional inducido $\mathrm{M}$ disminuye al aumentar el valor de $\mathrm{Ra}_{\mathrm{b}}{ }^{*}$, como puede deducirse de la Figura 5. Por lo tanto no puede inferirse a priori una relación directa entre el aumento del ancho del canal b y el flujo másico inducido $\mathrm{q}_{\mathrm{m}}$.

Sin embargo, para el rango de valores estudiados se observa que un aumento de la diferencia de temperaturas implica un aumento del caudal inducido.

En la Figura 9 se muestra el caudal inducido para el caso de una fachada en la que se ha fijado una altura de $3 \mathrm{~m}$ para diferentes valores de la diferencia de temperaturas y del ancho del canal. Se observa que, en todo el rango de los valores del ancho del canal, un aumento de la diferencia de temperaturas implica un aumento del caudal inducido. Asimismo y congruentemente con los resultados anteriores, se observa que el caudal máximo se obtiene para un ancho de canal de $10 \mathrm{~cm}$ hasta una diferencia de temperaturas de $20^{\circ} \mathrm{C}$. A partir de ese valor, el flujo máximo ocurre para un ancho de canal de $8 \mathrm{~cm}$.

\section{CONCLUSIONES}

En el presente trabajo se ha estudiado el fenómeno físico"efecto chimenea"que tiene lugar en la cámara de las fachadas venti-

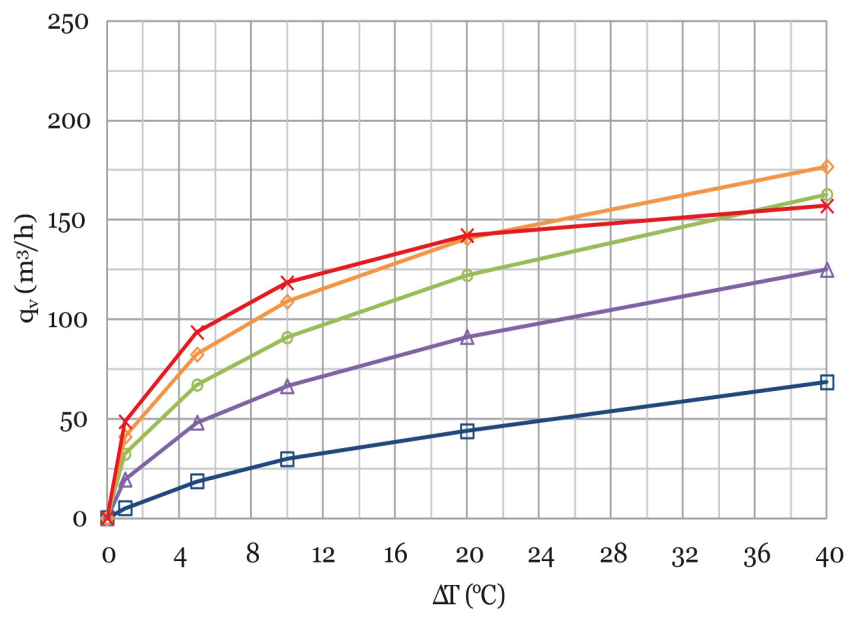

$\square \mathrm{b}=2 \mathrm{~cm} \triangle \mathrm{b}=4 \mathrm{~cm} \multimap \mathrm{b}=6 \mathrm{~cm} \leadsto \mathrm{b}=8 \mathrm{~cm} \leadsto \mathrm{b}=10 \mathrm{~cm}$

Figura 9. Caudal volumétrico de aire inducido en función de la diferencia de temperaturas para diferentes anchos de canal $(\mathrm{L}=3 \mathrm{~m})$.

ladas opacas. Se propone una correlación útil para el cálculo del flujo másico inducido en función del número de Rayleigh modificado $\mathrm{Ra}_{\mathrm{b}}{ }^{*}$. En este número adimensional quedan condensados tanto los parámetros geométricos (alto y ancho del canal) como la diferencia de temperaturas entre el aire exterior y el aire de las superficies del canal. El conjunto de casos de aplicación de las correlaciones cubre el rango habitual de interés en fachadas ventiladas opacas dentro del régimen laminar. Dado que en el parámetro $\mathrm{Ra}_{\mathrm{b}}{ }^{*}$ se condensan las tres variables de estudio (ancho de la fachada ventilada, alto de la fachada ventilada y diferencia de temperaturas), la correlación es aplicable a cualquier combinación de los mismos siempre y cuando se cumpla que el valor de $\mathrm{Ra}_{\mathrm{b}}{ }^{*}$ sea inferior a $10^{5}$. Típicamente, el campo de aplicación cubre casos de anchos de canal de hasta $10 \mathrm{~cm}$, diferencias de temperatura de hasta $40^{\circ} \mathrm{C}$ y cualquier altura del canal.

De los resultados presentados pueden estimarse los órdenes de magnitud del caudal inducido esperable en una fachada ventilada dada. Así, para una fachada ventilada de $3 \mathrm{~m}$ de altura (aproximadamente la altura de una planta), puede alcanzarse como máximo hasta un caudal inducido de $175 \mathrm{~m}^{3} / \mathrm{h}$ por metro de profundidad de la fachada, (caso con $8 \mathrm{~cm}$ de ancho de canal y $40^{\circ} \mathrm{C}$ de diferencia de temperaturas). Por el contrario, para diferencias de temperatura del orden de sólo $1^{\circ} \mathrm{C}$, el caudal inducido se reduce hasta los $50 \mathrm{~m}^{3} / \mathrm{h}$ por metro de profundidad (en el caso más favorable con $10 \mathrm{~cm}$ de ancho de canal).

Se ha realizado asimismo un análisis de sensibilidad de los parámetros que influyen en el flujo inducido en la fachada ventilada $\mathrm{q}_{\mathrm{v}}\left(\mathrm{m}^{3} / \mathrm{h}\right)$. Los resultados de dicho análisis se resumen en la Tabla 4.

Finalmente, del análisis de sensibilidad de los parámetros se pueden obtener las siguientes conclusiones:

Fijadas la altura y la diferencia de temperaturas, el caudal inducido presenta un valor máximo para un determinado valor del ancho del canal b. En el caso de fachadas ventiladas de $3 \mathrm{~m}$ de altura, para valores de diferencia de temperaturas de hasta $10^{\circ} \mathrm{C}$, un aumento del ancho del canal tiene asociado un aumento del caudal inducido en todo el rango de valores 
Tabla 4. Resumen de los resultados obtenidos del flujo inducido $\mathrm{q}_{\mathrm{v}}\left(\mathrm{m}^{3} / \mathrm{h}\right)$.

\begin{tabular}{|c|c|c|c|c|c|c|}
\hline$q_{v}\left(m^{3} / h\right)$ & $\mathbf{b}(\mathbf{c m})$ & $\mathrm{DT}=\mathbf{1}^{\circ} \mathrm{C}$ & $\mathrm{DT}=5^{\circ} \mathrm{C}$ & $\mathrm{DT}=10^{\circ} \mathrm{C}$ & $\mathrm{DT}=\mathbf{2 0}^{\circ} \mathrm{C}$ & $\mathrm{DT}=40^{\circ} \mathrm{C}$ \\
\hline \multirow{5}{*}{$\mathrm{L}=3 \mathrm{~m}$} & 2 & 5,05 & 18,57 & 29,69 & 43,73 & 68,36 \\
\hline & 4 & 19,47 & 48,09 & 66,41 & 91,16 & 124,96 \\
\hline & 6 & 32,21 & 66,99 & 90,80 & 122,10 & 162,77 \\
\hline & 8 & 40,90 & 82,30 & 108,87 & 140,77 & 176,72 \\
\hline & 10 & 48,48 & 93,40 & 118,35 & 142,13 & 156,96 \\
\hline \multirow{5}{*}{$\mathrm{L}=6 \mathrm{~m}$} & 2 & 5,44 & 21,71 & 36,90 & 58,99 & 87,50 \\
\hline & 4 & 25,89 & 68,43 & 96,31 & 133,35 & 183,92 \\
\hline & 6 & 45,90 & 98,33 & 134,30 & 182,61 & 247,41 \\
\hline & 8 & 59,75 & 122,73 & 165,15 & 219,57 & 287,77 \\
\hline & 10 & 71,53 & 142,88 & 187,68 & 239,87 & 295,58 \\
\hline \multirow{5}{*}{$\mathrm{L}=9 \mathrm{~m}$} & 2 & 5,60 & 23,31 & 40,62 & 67,34 & 104,57 \\
\hline & 4 & 29,34 & 82,40 & 118,76 & 165,90 & 229,80 \\
\hline & 6 & 55,39 & 122,65 & 168,22 & 229,75 & 313,18 \\
\hline & 8 & 74,26 & 154,22 & 208,89 & 280,59 & 373,45 \\
\hline & 10 & 89,48 & 181,29 & 241,28 & 315,12 & 401,89 \\
\hline
\end{tabular}

de b considerado (hasta $10 \mathrm{~cm}$ ). Sin embargo, para diferencias de temperatura mayores, el máximo caudal inducido se obtiene para anchos de canal menores $(9 \mathrm{~cm}$ para una diferencia de temperaturas de $20{ }^{\circ} \mathrm{C}$ y $8 \mathrm{~cm}$ para $40{ }^{\circ} \mathrm{C}$ ).

Fijados el ancho y la diferencia de temperaturas, un aumento de la altura de la fachada ventilada implica un aumento en el flujo másico inducido.

Fijados el ancho y la altura de la fachada ventilada, el caudal inducido presenta un valor máximo para un determinado valor de la diferencia de temperaturas. Sin embargo, en el caso particular de fachadas ventiladas de $3 \mathrm{~m}$ de altura, un aumen- to de la diferencia de temperaturas implica un aumento del caudal inducido en todo el rango de diferencia de temperaturas estudiado (hasta $40{ }^{\circ} \mathrm{C}$ ).

\section{AGRADECIMIENTOS}

La realización de este estudio ha sido posible gracias a la financiación del proyecto I+D del Plan Nacional: «Integración de sistemas constructivos industrializables de fachadas ventiladas activas para el aseguramiento de un consumo energético eficiente y de la calidad de aire interior. Aplicación a edificios no residenciales», Ref. BIA2006-15398-Co4-04, del Ministerio de Educación y Ciencia.

\section{REFERENCIAS}

(1) Gagliano, A., Patania, F., Ferlito, A. Nocera, F., Galesi, A.. (2011). Computational Fluid Dynamic Simulations of Natural Convection in Ventilated Facades. En Amimul, A. (Ed.) Evaporation, Condensation and Heat transfer. Doi: http://dx.doi.org/10.5772/19817.

(2) Irulegi, O., Serra, A.,Hernández, R., Ruiz-Pardo, A., Torres, L. (2012). Fachadas ventiladas activas para reducir la demanda de calefacción en los edificios de oficinas. El caso de España. Informes de la Construcción, 64(528): 575-585, doi: http://dx.doi.org/10.3989/ic.11.099.

(3) Balocco, C. (2002). A simple model to study ventilated facades energy performance. Energy and Buildings, 34(5): 469-475, doi: http://dx.doi.org/10.1016/So378-7788(01)00130-X.

(4) Marinosci, C., Strachan, P., Semprini, G., Morini, G. L. (2011). Empirical validation and modelling of a naturally ventilated rainscreen facade building. Energy and Buildings, 43(4): 853-863, doi: http://dx.doi.org/10.1016/j.enbuild.2010.12.005.

(5) Ruiz-Pardo, A. (2008). Ahorro energético mediante el uso de elementos de doble envolvente transparente-opaco (Tesis Doctoral). Sevilla, España: Universidad de Sevilla.

(6) Suárez, Ch. (2013). Modelización CFD de los flujos de calor y masa en cámaras de aire. Aplicación al cálculo de coeficientes convectivos y flujos de aire en fachadas ventiladas (Tesis Doctoral). Sevilla, España: Universidad de Sevilla.

(7) Kheireddine, A.S., Houla Sanda, M., Chaturvedi, S.K., Mohieidin, T.O. (1997). Numerical Prediction Of Pressure Loss Coefficient And Induced Mass Flux For Laminar Natural Convective Flow In A Vertical Channel. Energy 22(4): 413-423, doi: http://dx.doi.org/10.1016/0360-5442(95)00060-7.

(8) Kettleborough, C.F. (1972). Transient Laminar Free Convection Between Heated Vertical Plates Including Entrance effects. Int. J. Heat Mass Transfer, 15(5): 883-896, doi: http://dx.doi.org/10.1016/o017-9310(72)90228-1.

(9) Nakamura, H., Asako, Y., Naitou, T. (1982). Heat Transfer by Free Convection Between Two Parallel Flat Plates. Numer. Heat Transfer 5(1): 95-106, doi: http://dx.doi.org/10.1080/10407788208913437.

(10) Naylor, D., Floryan, J. M., Tarasuk, J. D. (1991). A Numerical Study of Developing Free Convection Between Isothermal Vertical Plates. ASME J. Heat Transfer, 113(3): 620-626, doi: http://dx.doi.org/10.1115/1.2910610.

(11) Chang, T. S., Lin, T. F. (1989). Transient Buoyancy-Induced Flow Through a Heated, Vertical Channel of Finite Height. Numer. Heat Transfer, 16 (1) 15-35, doi: http://dx.doi.org/10.1080/10407788908944704.

(12) Ramanathan, S., Kumar, R. (1991). Correlations for Natural Convection Between Heated Vertical Plates. ASME J. Heat Transfer, 113(1): 97-107, doi: http://dx.doi.org/10.1115/1.2910557. 
(13) Shyy, W., Gingrich, W. K., Gebhart, B. (1992). Adaptive Grid Solution for Buoyancy-Induced Flow in Vertical Slots. Numer. Heat Transfer. Part A, 22(1): 51-70, doi: http://dx.doi.org/10.1080/10407789208944758.

(14) Morrone, B., Campo, A., Manca, O. (1997). Optimum Plate Separation in Vertical Parallel-Plate Channels for Natural Convective Flows: Incorporation of Large Spaces at the Channel Extremes. Int. J. Heat Mass Transfer, 40(5): 993-1000, doi: http://dx.doi.org/10.1016/o017-9310(96)o0197-4.

(15) Campo, A., Manca, O., Morrone, B. (1999). Numerical Analysis of Partially Heated Vertical Parallel Plates in Natural Convective Cooling. Numer. Heat Transfer, Part A, 36(2): 129-151, doi: http://dx.doi.org/ 10.1080/104077899274813.

(16) Ayinde, T. F., Said, S. A. M., Habib, M. A. (2006). Experimental investigation of turbulent natural convection flow in a channel. Heat Mass Transfer, 42(3): 169-177, doi: http://dx.doi.org/10.1007/s00231-005-0017-2.

(17) Carl-Olof Olsson. (2004). Prediction of Nusselt Number and Flow Rate of Buoyancy Driven Flow Between Vertical Parallel Plates. Transactions of the ASME. Serie C: Journal of heat transfer, 126(1): 97-104, doi: http://dx.doi. org/10.1115/1.1643908. 